seem to be homing. Heitzmann (ibid., 114) arrives at $35-40,000$ on similar grounds. If this is correct, it follows that some 80 per cent of the total protein of the rod outer segment is rhodopsin.

\section{NUCLEIC ACIDS \\ Modfification Methylase}

from our Cell Biology Correspondent

THE problem faced by organisms of distinguishing self from non-self has, of course, led to the emergence and diversification of the immune system; it has also resulted in the evolution in bacteria, and perhaps elsewhere, of host restriction and modification systems which prove to be sets of enzymes capable of marking and distinguishing indigenous DNA from invading DNA. Since their discovery the question of how bacterial modification and restriction enzymes work has attracted much attention and, thanks to the efforts of several research groups, there is now a clear overall picture of the process.

It seems that, in any particular strain of bacteria, DNA after replication is modified by the methylation of a quite small number of bases at specific sites in the molecule. A specific modification methylase catalyses the reaction and there is ample evidence which indicates that it uses $\mathrm{S}$-adenosylmethionine as a source of methyl groups. As a result of its methylation the DNA is rendered resistant to the cell's restriction endonuclease, most probably because the methyl groups mask the sites in the DNA which the endonuclease specifically attacks. By contrast, invading DNA from another strain, either unmodified or modified to a different pattern, is unprotected and therefore is accessible to the restriction endonuclease which degrades it. Modification and restriction enzymes, in short, provide the bacterial cell with a defence against invading genomes.

Two reports in the Journal of Molecular Biology $(63,1,9 ; 1972)$ by Smith. Arber and Kühnlein and Kühnlein and Arber leave little doubt as to the correctness of this overall picture and add some fine detail to it. Exploiting the fact that the single stranded DNA genome of coliphage fd contains very few methylated bases, Smith et al. have succeeded in measuring, by two methods, the number of N-6-methyladenine residues per genome in unmodified phage and modified phage grown on Escherichia coli B. They find, gratifyingly, twice as many methyladenine residues in the modified DNA as in the unmodified DNA; furthermore, when a mutant fd phage which is insensitive to $B$ restriction is grown on $E$. coli B its DNA is not methylated at the $\mathrm{B}$ modification site and so contains only half the number of methyladenine residues present in $\mathbf{B}$ modified wild type phage. The residual methyladenine residues in the mutant DNA presumably have no relation to the $B$ modification restriction process. In short, B modification involves methylation of DNA and in the case of fd DNA complete B modification results in the methylation of one adenine residue at each of two B specific modification sites.

Paralleling these measurements Kühnlein and Arber have continued their investigations of partially purified $E$. coli B modification methylase, an enzyme which they have shown methylates in vitro double stranded replicative form fd DNA but not single stranded fd genomes. Methylation of replicative form DNA in vitro follows first order kinetics-all sites are equally efficient methyl group acceptors-and ceases when each replicative form molecule is saturated with four methyl groups. Moreover, the replicative form DNA of a mutant fd phage less sensitive in vivo to $B$ restriction than wild type phage is saturated in vitro by two methyl groups and the replicative form of a double mutant, insensitive to $B$ restriction, does not act as a substrate for the modification methylase. These findings, as well as supporting those of Smith et al. indicate that the $B$ specific modification sites on both the plus and minus strands of fd DNA are methylated at equal rates and probably have symmetric base sequences, a situation for which the modification restriction system of Haemophilus influenzae provides a precedent.

That such a small change as the methylation of two adenine residues in an fd DNA chain of some 6,600 bases renders the DNA resistant to a restriction endonuclease indicates the high specificity of these nucleolytic enzymes, a specificity which will no doubt prove useful. Danna and Nathans (Proc. US Nat. Acad. Sci., 68, 2913; 1971), for example, have used $H$. influenzae restriction enzyme to cleave form I DNA of simian virus 40 , a closed, circular double stranded DNA, into eleven resolvable fragments eight of which are yielded in amounts equimolar to the original DNA.

As Danna and Nathans comment, with so many restriction enzymes available, and more no doubt awaiting discovery, it may well be possible by judicious selection of enzymes to cut the genome of small DNA viruses, such as SV40 or polyoma virus, into a set of overlapping fragments small enough for an attempt to be made to sequence them. In other words the analysis of the base sequence of a DNA tumour virus genome may be one of the bonuses of investigations of bacterial modification-restriction systems.

\title{
DNA Complementary to Globin mRNA
}

IN next Wednesday's Nature New Biology (February 9) there are reports by Baltimore's group and Spiegelman's group of the synthesis by reverse transcriptase isolated from avian myeloblastosis virus (the most convenient source of a reverse transcriptase) of a single stranded DNA molecule complementary to at least part of the messenger RNAs for rabbit and human globins. One of the first properties of reverse transcriptase to be discovered was its lack of rigorous template specificity; unlike the RNA replicases which replicate the genomes of the RNA bacteriophages and have extremely fastidious template requirements, reverse transcriptases are capable of using a variety of natural and synthetic RNAs as templates for DNA synthesis.

This finding immediately raised the possibility of using reverse transcriptase to make radioactively labelled DNAs complementary to specific messenger RNAs. Such DNA molecules, of high specific activity, might then be used as a probe for genes and gene products in nucleated cells. Globin messenger RNAs from various species, which have been partially purified, provide the obvious model system in which to test these ideas and several groups of re- searchers began the obvious experiments. As Verma, Temple, Fan and Baltimore and Kacian et al. are first to report, in the presence of short poly(dT) chains, which presumably act as primers, and in the presence of actinomycin D to restrict DNA synthesis to single strands, reverse transcriptase from avian myeloblastosis virus will indeed synthesize an $8 \mathrm{~S}$ single stranded DNA when programmed with $10 \mathrm{~S}$ globin messenger preparations from rabbit or human red cells. Furthermore, although, as Baltimore's group observe, it has yet to be rigorously proven that it is the active globin messenger molecules that are serving as templates for this DNA, a variety of lines of evidence indicate that this is most probably the case.

In short, even if reverse transcriptase proves to be of little significance to cancer researchers out to cure or prevent that disease, it seems destined to prove an extremely useful reagent for molecular biologists faced with the task of unravelling the genome and mechanisms which regulate its expression in eukaryotic cells. To be able to make very hot DNA complementary to specific messenger RNAs is something of a pipe dream come true. 